\title{
Safety effects of work zone advisory systems under the intelligent connected vehicle environment: a microsimulation approach
}

\author{
Suyi Mao, Guiming Xiao and Faeyoung Lee \\ School of Traffic and Transportation Engineering, Central South University, Changsha, China \\ Ling Wang \\ College of Transportation Engineering, Tongji University, Shanghai, China \\ Zijin Wang \\ Department of Civil, Environmental and Construction Engineering, University of Central Florida, Orlando, Florida, USA, and \\ Helai Huang \\ School of Traffic and Transportation Engineering, Central South University, Changsha, China
}

\begin{abstract}
Purpose - This study aims to investigate the safety effects of work zone advisory systems. The traditional system includes a dynamic message sign (DMS), whereas the advanced system includes an in-vehicle work zone warning application under the connected vehicle (CV) environment.

Design/methodology/approach - A comparative analysis was conducted based on the microsimulation experiments.

Findings - The results indicate that the CV-based warning system outperforms the DMS. From this study, the optimal distances of placing a DMS varies according to different traffic conditions. Nevertheless, negative influence of excessive distance DMS placed from the work zone would be more obvious when there is heavier traffic volume. Thus, it is recommended that the optimal distance DMS placed from the work zone should be shortened if there is a traffic congestion. It was also revealed that higher market penetration rate of CVs will lead to safer network under good traffic conditions.

Research limitations/implications - Because this study used only microsimulation, the results do not reflect the real-world drivers' reactions to DMS and CV warning messages. A series of driving simulator experiments need to be conducted to capture the real driving behaviors so as to investigate the unresolved-related issues. Human machine interface needs be used to simulate the process of in-vehicle warning information delivery. The validation of the simulation model was not conducted because of the data limitation.

Practical implications - It suggests for the optimal DMS placement for improving the overall efficiency and safety under the CV environment. Originality/value - A traffic network evaluation method considering both efficiency and safety is proposed by applying traffic simulation.
\end{abstract}

Keywords Connected vehicles, Driver behaviors and assistance, Intelligent vehicles, Vehicle dynamics and control, Vehicle-to-infrastructure communication (V2I), Work zone, Dynamic message sign (DMS), In-vehicle warning, Traffic simulation

Paper type Research paper

\section{Introduction}

Highway maintenances are essential to keep operational efficiency and safety. During the maintenance, the highway capacity significantly decreases and the crash risk increases. Regular and irregular highway maintenances are essential to keep operational efficiency and safety. In developing countries, there are many highway constructions because of the rapid increases in travel and freight demands. Nevertheless, during the maintenance and construction phases, the highway capacity significantly decreases, whereas the crash risk increases. In the USA, 809 people were killed at work zones in 2017 (Figure 1).

The current issue and full text archive of this journal is available on Emerald Insight at: https://www.emerald.com/insight/2399-9802.htm

Journal of Intelligent and Connected Vehicles 4/1 (2021) 16-27

Emerald Publishing Limited [ISSN 2399-9802] [DOI 10.1108/JICV-07-2020-0006]
Compared to the fatalities in 2010, the number of work zone fatalities has increased by $38 \%$. In particular, the numbers of truck- and pedestrian-involved work zone fatalities have increased by $99 \%$ and $69 \%$, respectively, from 2010 to 2017 . The number of work zone workers who were killed in traffic

(C) Suyi Mao, Guiming Xiao, Jaeyoung Lee, Ling Wang, Zijin Wang and Helai Huang. Published in fournal of Intelligent and Connected Vehicles. Published by Emerald Publishing Limited. This article is published under the Creative Commons Attribution (CC BY 4.0) licence. Anyone may reproduce, distribute, translate and create derivative works of this article (for both commercial and non-commercial purposes), subject to full attribution to the original publication and authors. The full terms of this licence maybe seen at http://creativecommons.org/licences/by/4.0/ legalcode

This study was funded by National Key R\&D Program of China (2020YFB1600400), Innovation-Driven Project of Central South University (2020CX013) and Shanghai Sailing Program (19YF1451300).

Received 16 July 2020

Revised 13 December 2020

Accepted 5 February 2021 
Figure 1 Traffic fatalities at work zones in the USA (2010-2017)

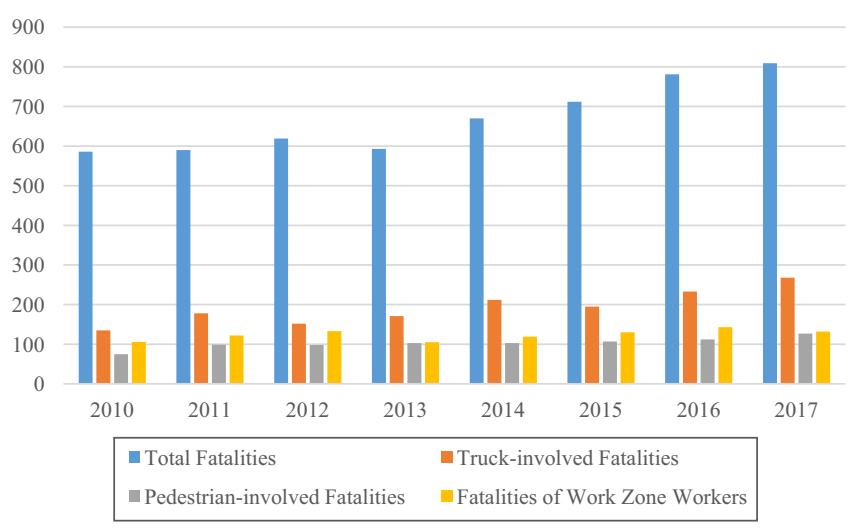

crashes were 106 and 132, respectively, in 2010 and 2017 (National Work Zone Safety Information Clearinghouse, 2019). The statistics indicate that traffic safety at work zones is getting worse, and safety researchers should provide effective solutions to enhance safety at work zones.

There have been many practices and research studies suggesting ways to improve traffic safety at work zones. One of the most common countermeasures is to warn drivers of the presence of a work zone. A DMS, which has been widely used for traffic control at work zones, was proven to effectively influence driver behavior (Dudek, 2004; Strawderman et al., 2013). However, to ensure DMS can work successfully, drivers must notice the messages of DMS and obey the control advice (Schofer et al., 1993; Hassan et al., 2012; Jones and Thompson, 2003). With the development of advanced information technologies, internet of vehicles is becoming a reality. In the connected vehicle (CV) environment, work zone advisory systems are more advanced than traditional ones by using roadside devices to delivery traffic information. Using these technologies, accurate information can be extracted and provided to drivers to alert them to present danger or assist in vehicle control to help drivers make optimal decisions at work zones (Olia et al., 2013; Genders and Razavi, 2016; Abdulsattar et al., 2018). To improve the safety in the work zone, more accurate and detailed traffic information should be sent to drivers through the DMSs or through the CV communication technologies (Teizer et al., 2010; Blackman et al., 2014). Nevertheless, the previous studies have only focused on existing intelligent transportation system (ITS) applications or solely CV technology applications, separately. Until the market penetration rate becomes sufficiently high, essential safety and traffic information through the existing ITS technologies should be provided for manually driven vehicles. Therefore, it is necessary to study how to maximize the efficiency and safety of highway work zone when CVs and manually driven vehicles are mixed in the road network.

The main objective of this study is to investigate the coordinated optimization strategies for existing ITSs and upcoming CV technologies. In this paper, microsimulation software VISSIM is used to design different experimental scenarios. Different market penetration rates, different distance between DMS and work zone, different traffic volumes and different drivers' compliance have been considered. The rest of the paper is organized as follows. The experimental design section explains the main idea of the research and the result section summarizes the findings. Subsequently, the discussion section explains and interprets the findings. Finally, conclusions are drawn in the conclusion section.

\section{Methodology}

In this study, microsimulation was applied to achieve the abovementioned objectives. Microsimulation has been popularly used for evaluating effectiveness of safety of ITS and CV applications at network level (Wang et al., 2017; Rahman et al., 2018). Different case-controlled experiments were designed by using microsimulation software VISSIM.

An $8 \mathrm{~km}$, three-lane highway was built in VISSIM. The speed limit is $120 \mathrm{~km} / \mathrm{h}$, and the lane width is $3.75 \mathrm{~m}$. The length of work zone is $500 \mathrm{~m}$. The distances between DMS and the work zone are different by scenarios. Thus, eight different distances (i.e. $400 \mathrm{~m}, 600 \mathrm{~m}, 1,000 \mathrm{~m}, 1,400 \mathrm{~m}$, $1,800 \mathrm{~m}, 2,200 \mathrm{~m}, 2,600 \mathrm{~m}$ and 3,000 m) were considered. Two data collection points were installed at $500 \mathrm{~m}$ from the upstream of DMS and the downstream of the work zone, and it is worth mentioning that the length of the data collection segment is different in different scenarios according to the research objectives. Average travel time, average speed, average queue time and average delay time of all vehicles were considered as the evaluation efficiency indicators and the number of time to collision (TTC) values less than $1.5 \mathrm{~s}$ was considered as the safety evaluation indicator.

The formula of $T T_{\text {mean }}$ is specified as follows:

$$
T T_{\text {mean }}=\frac{\sum_{i=1}^{N} T T_{i}}{N}
$$

where $T T_{\text {mean }}$ is the average travel time of the data collection segment; $i$ is the number of vehicle; $T T_{i}$ is the travel time of a vehicle numbered $i$; and $N$ is the total number of vehicles.

The formula of $V_{\text {mean }}$ is specified as follows:

$$
V_{\text {mean }}=\frac{\sum_{i=1}^{N} V_{i}}{N}
$$

where $V_{\text {mean }}$ is the average speed of the data collection segment; $i$ is the number of vehicle; $V_{i}$ is the average speed of a vehicle numbered $i$; and $N$ is the total number of vehicles.

The calculation formula of $Q T_{\text {mean }}$ is specified as follows:

$$
Q T_{\text {mean }}=\frac{\sum_{i=1}^{N} Q T_{i}}{N}
$$

where $Q T_{\text {mean }}$ is the average queue time of the data collection segment; $i$ is the number of vehicle; $Q T_{i}$ is the average queue time of a vehicle numbered $i$; and $N$ is the total number of vehicles.

The calculation formula of $D T_{\text {mean }}$ is specified as follows: 


$$
D T_{\text {mean }}=\frac{\sum_{i=1}^{N} D T_{i}}{N}
$$

where $D T_{\text {mean }}$ is the average delay time of the data collection segment; $i$ is the number of vehicle; $D T_{i}$ is the average delay time of a vehicle numbered $i$; and $N$ is the total number of vehicles.

The calculation formula of $T T C_{i m p r o v e d}$ is specified as follows:

$$
T T C_{\text {improved }}=\frac{d_{L F}}{v_{F-} v_{L}}
$$

where $d_{L F}$ is the distance between the leading and following vehicle; $v_{F}$ is velocity of the following vehicle; and $v_{L}$ is velocity of the leading vehicle. $T T C_{\text {improved }}$ is computed for a leading vehicle and following vehicle, with the potential incident being a rear-end collision. The value of $T T C_{\text {improved }}$ at less than a threshold value of $1.5 \mathrm{~s}$ is defined as $N_{T T C \leq 1.5}$, which was considered indicative of two vehicles exhibiting a high probability of colliding (Van der Horst and Hogema, 1993). Because microsimulation software lacks physical collision modeling, researchers usually use less-than-ideal methods to evaluate safety. Hence, the surrogate safety measurement $T T C_{\text {improved }}$ was chosen to identify potential of one of the rearend collisions to approximate network safety. Experiments were designed, respectively, to represent different simulation scenarios of traditional and advanced approaches (Figure 2).

According to previous studies, it is difficult to simulate drivers' response when they observe DMS. Drivers' compliance rate in different DMS distance scenarios is inconsistent. Many researchers have put efforts in studying this topic (Ardeshiri and Jeihani, 2014; Brewer et al., 2006). Some researchers conducted a survey questionnaire to investigate drivers' compliance rate in different driving scenarios (Debnath et al., 2015; Weng and Meng, 2012; Mohammadi et al., 2011). However, some literatures found that drivers' compliance rate abstracted from the results of the questionnaire survey results was higher than real drivers' compliance rate in most cases, which shows that survey-based compliance rates are not very convincing enough (Lajunen and Summala, 2003; Wåhlberg et al., 2011). To better simulate real driving scenarios, some researchers used driving simulators to study the compliance rate (Rahman et al., 2017; Whitmire et al., 2011; Bella, 2005; Shakouri et al., 2014). Nevertheless, other studies pointed out that there are still errors when using driving simulators. Because the number of volunteers who participated in the driving simulator experiment is limited, the results of driving simulator experiments cannot realistically reflect the influence of surrounding traffic environment on drivers' behaviors (Carsten and Jamson, 2011; Koutsopoulos et al., 1995; Olson et al., 2009).

In this paper, a new approach based on VISSIM and genetic algorithm (GA) was proposed to simulate the drivers' behaviors when they are passing through the work zone. According to traffic equalization principle, in the beginning, every driver in the merge lane (the lane with work zone) tries to shorten his/her travel time by merging into the through lane at different positions in the upstream. This process will carry on until all the drivers have the same travel time. Hence, a reasonable assumption was proposed, which is vehicles in the merge lane in the upstream of work zone merge at different positions, where for every vehicle, their travel times are approximately the same. Because in VISSIM we cannot set the merging point consistently, we divided the interval between DMS and work zone into several subintervals of $200 \mathrm{~m}$ to represent different merging positions. By setting multiple connectors and partial paths, different subintervals correspond to different initial merging positions. If the vehicle passes through the partial route decision point, it then starts to look for the opportunity to change lanes. If there is no opportunity temporarily, it will continue to move forward until finds the right lane change opportunity. Each subinterval corresponds to a lane change ratio, which stands for the number of drivers choosing to change lane here.

Therefore, this implies that under optimized lane-changing ratios setting, travel time of every subinterval should be approximately the same. However, lane-changing ratios of each subinterval are not easy to calculate. This new approach was used to obtain the approximate global optimal solution. GA was used to generate the initial population and complete the process of selection and variation. Initial population size is 10 . Selection process uses the roulette rule. Mutation probability is 0.05 . The number of iterations was 50 generations. MATLAB codes are used to call VISSIM's COM component function. Travel time data of every vehicle in the data collection segment is collected and variance is calculated, which is the fitness index of population. Three different traffic volume scenarios are simulated (i.e. 3,000 vehicles per hour [vph], 5,000 vph and $10,000 \mathrm{vph}$ ), which represent different traffic volume conditions. Because a single simulation might suffer instability, each experiment was simulated by setting different random seeds for ensuring the accuracy and stability of the simulation results. The final experimental results are summarized in the Figures 3-5 (Tables 1-3).

Figure 2 Schematic of experimental design

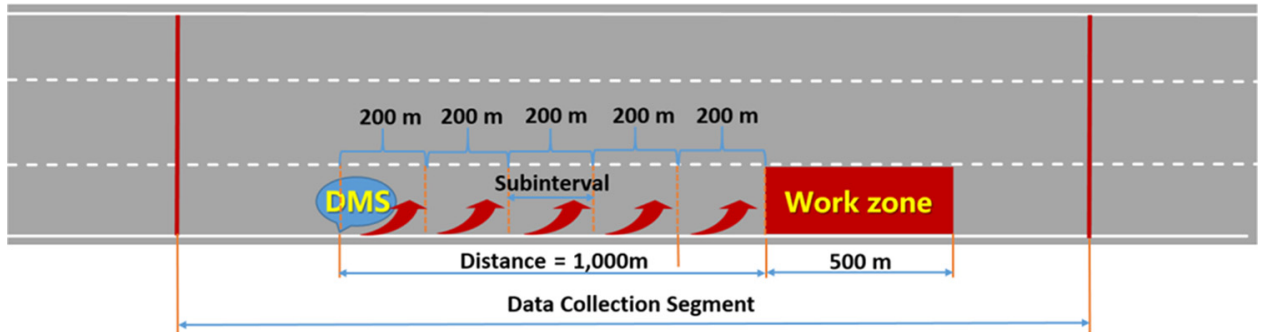


Figure 3 Optimal lane change ratios by different DMS distance (3,000 vph)

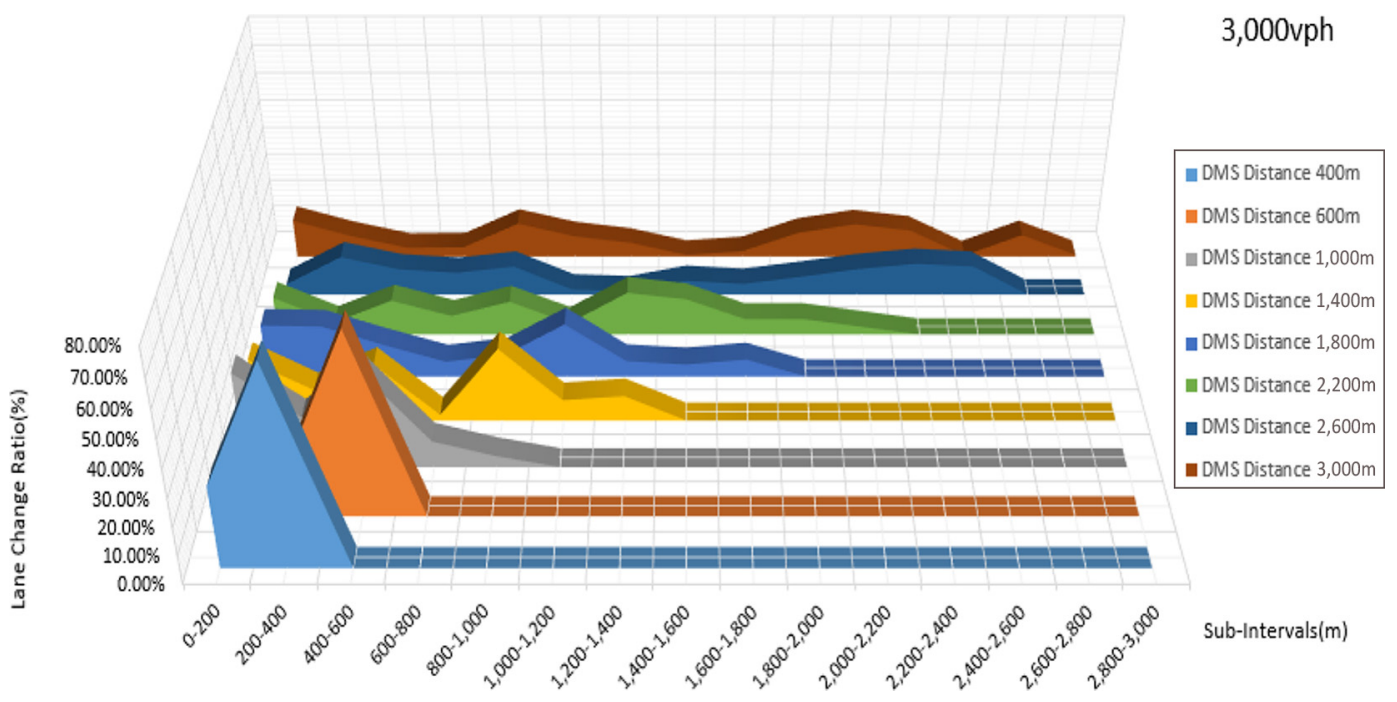

Figure 4 Optimal lane change ratios by different DMS distance (5,000 vph)

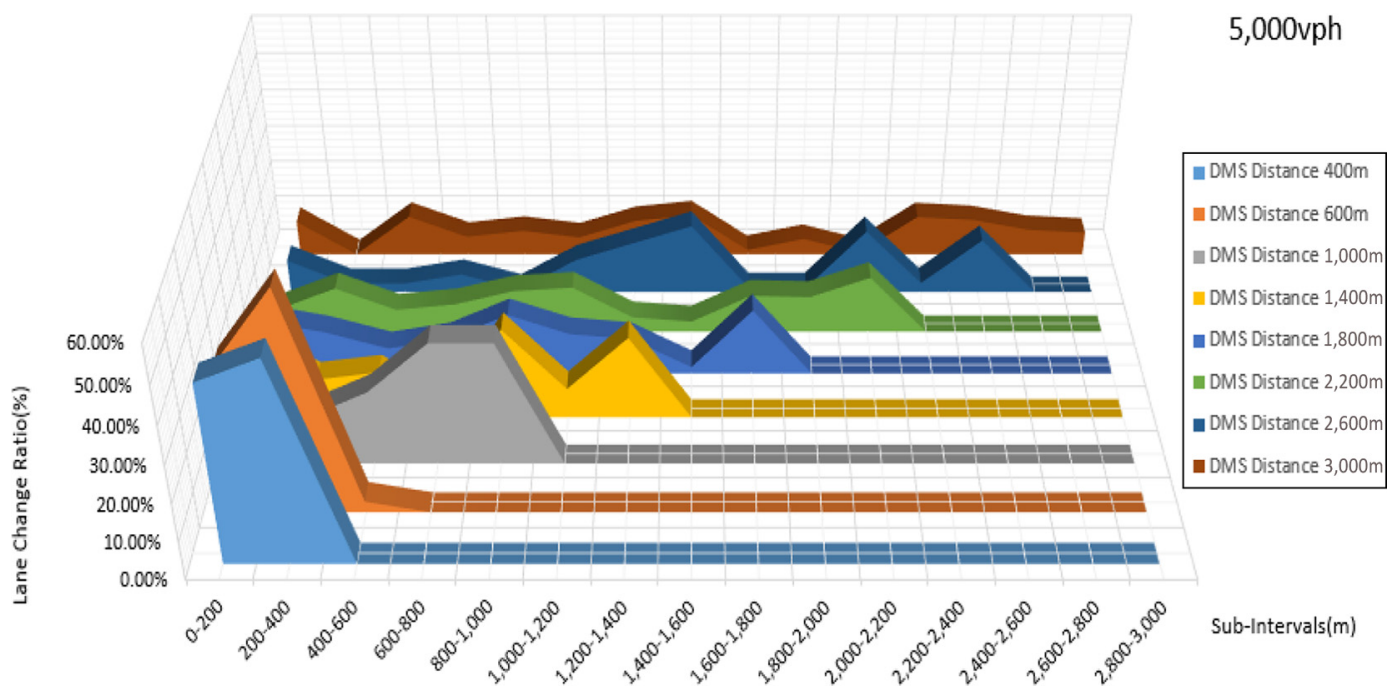

It is observed from the experimental results that as the DMS distance from the work zone becomes longer, the lane change ratio of different subinterval becomes more evenly. It might infer that if there are more subintervals, the wider the range of lane change options for drivers would be available. To find a path with a shorter travel time, drivers may avoid changing lanes in a crowded place. Furthermore, it is not hard to find that the longer the DMS distance is, the further the subinterval with maximum lane change ratio is from the work zone. This is because, the closer the work zone, the higher the possibility of time spent on changing lanes. Therefore, when there is a sufficient distance gap for changing lanes, drivers will change lanes in advance as soon as possible. But under different traffic volume conditions, the situation is different. As the traffic flow increases, there are fewer opportunities to change lanes away from the work zone. Hence, when there is a traffic congestion, the sum of lane change ratio including subintervals that far from the work zone is smaller. However, GA is not the algorithm to find the global optimal solution. The optimal lane change ratio that makes travel time variance the least may not be the optimal solution. But the approach used in this paper can explain the drivers' lane change behaviors from the perspective of the whole transportation system, which is closer to the real-world driving behaviors. The experimental results of optimal lane change ratio are used to operate simulation when evaluating the safety and efficiency of work zone advisory systems.

Experiment 1: In the traditional environment without $\mathrm{CV}$, $T T_{\text {mean }}, V_{\text {mean }}, Q T_{\text {mean }}, D T_{\text {mean }}$ and $T T C_{\text {improved }}$ varied in different traffic volume and different DMS distance scenarios. DMS is a traditional traffic information delivery approach. According to the experimental results of optimal lane change ratio, different simulation experiments representing different DMS distance scenarios are conducted. Three types of traffic 
Figure 5 Optimal lane change ratios by different DMS distance (10,000 vph)

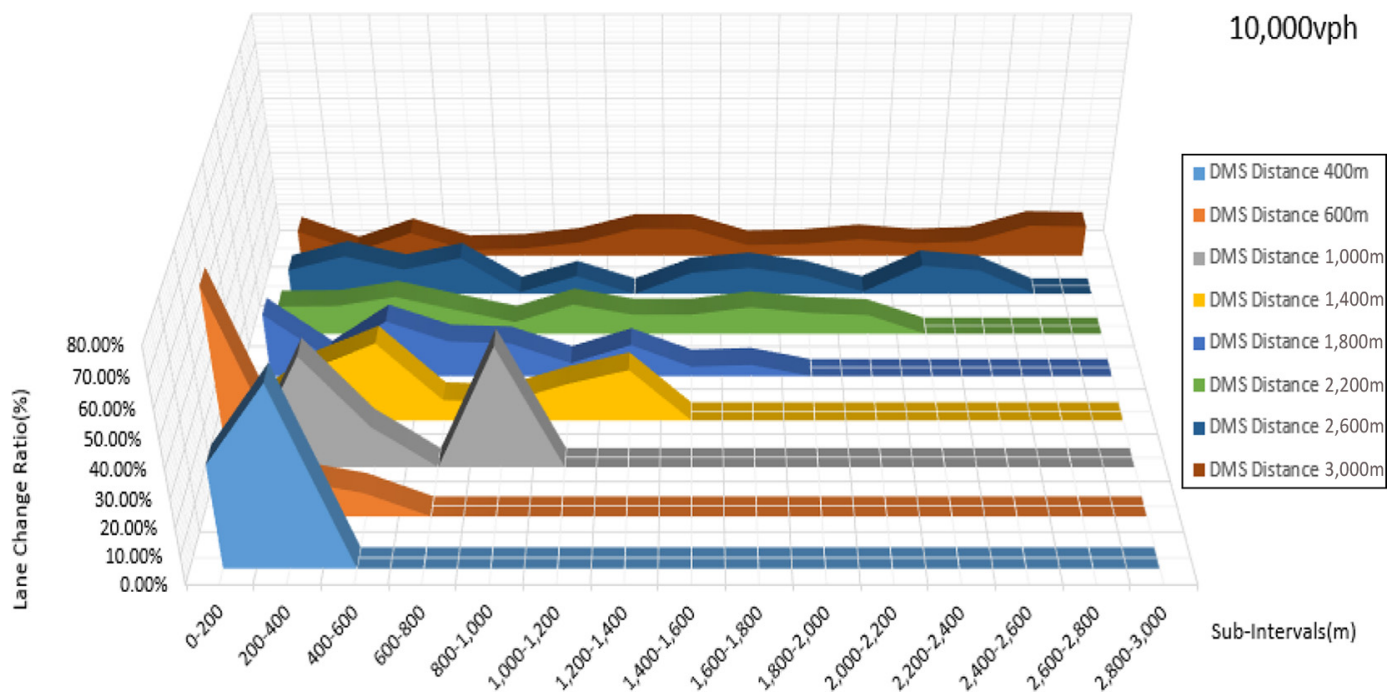

Table 1 Optimal lane change ratios by different DMS distance (3,000 vph)

\begin{tabular}{|c|c|c|c|c|c|c|c|c|c|c|c|c|c|c|c|}
\hline \multirow{2}{*}{$\frac{\text { DMS distance }(\mathrm{m})}{400}$} & \multicolumn{15}{|c|}{ Lane change ratio of subintervals (\%) } \\
\hline & 29 & 71 & & & & & & & & & & & & & \\
\hline 600 & 14 & 21 & 65 & & & & & & & & & & & & \\
\hline 1,000 & 34 & 18 & 34 & 10 & 4 & & & & & & & & & & \\
\hline 1,400 & 23 & 11 & 21 & 2 & 26 & 8 & 9 & & & & & & & & \\
\hline 1,800 & 19 & 19 & 12 & 6 & 8 & 20 & 6 & 4 & 6 & & & & & & \\
\hline 2,200 & 14 & 5 & 13 & 7 & 13 & 4 & 16 & 13 & 6 & 6 & 3 & & & & \\
\hline 2,600 & 4 & 14 & 10 & 9 & 11 & 2 & 1 & 5 & 4 & 7 & 10 & 12 & 11 & & \\
\hline 3,000 & 14 & 8 & 3 & 4 & 13 & 8 & 5 & 1 & 2 & 9 & 13 & 10 & 1 & 8 & 1 \\
\hline
\end{tabular}

Table 2 Optimal lane change ratios by different DMS distance (5,000 vph)

\begin{tabular}{|c|c|c|c|c|c|c|c|c|c|c|c|c|c|c|c|}
\hline \multirow{2}{*}{$\begin{array}{l}\text { DMS distance }(\mathrm{m}) \\
400\end{array}$} & \multicolumn{15}{|c|}{ Lane change ratio of subintervals (\%) } \\
\hline & 47 & 53 & & & & & & & & & & & & & \\
\hline 600 & 39 & 58 & 3 & & & & & & & & & & & & \\
\hline 1,000 & 3 & 13 & 20 & 32 & 32 & & & & & & & & & & \\
\hline 1,400 & 20 & 11 & 13 & 2 & 24 & 8 & 22 & & & & & & & & \\
\hline 1,800 & 15 & 11 & 7 & 10 & 16 & 11 & 10 & 2 & 18 & & & & & & \\
\hline 2,200 & 7 & 12 & 6 & 8 & 12 & 13 & 4 & 3 & 10 & 10 & 15 & & & & \\
\hline 2,600 & 9 & 3 & 3 & 5 & 1 & 9 & 14 & 19 & 1 & 1 & 17 & 3 & 15 & & \\
\hline 3,000 & 10 & 0 & 11 & 5 & 7 & 5 & 10 & 11 & 1 & 5 & 1 & 11 & 10 & 7 & 6 \\
\hline
\end{tabular}

Table 3 Optimal lane change ratios by different DMS distance (10,000 vph)

\begin{tabular}{|c|c|c|c|c|c|c|c|c|c|c|c|c|c|c|c|}
\hline DMS distance (m) & & & & & & Lane & ge $r$ & of su & ervé & & & & & & \\
\hline 400 & 36 & 64 & & & & & & & & & & & & & \\
\hline 600 & 78 & 13 & 9 & & & & & & & & & & & & \\
\hline 1,000 & 2 & 40 & 15 & 0 & 43 & & & & & & & & & & \\
\hline 1,400 & 6 & 20 & 28 & 7 & 7 & 13 & 19 & & & & & & & & \\
\hline 1,800 & 23 & 7 & 20 & 13 & 12 & 5 & 12 & 4 & 4 & & & & & & \\
\hline 2,200 & 11 & 11 & 14 & 9 & 5 & 11 & 7 & 7 & 10 & 8 & 7 & & & & \\
\hline 2,600 & 9 & 14 & 9 & 14 & 1 & 7 & 0 & 8 & 10 & 7 & 1 & 11 & 9 & & \\
\hline 3,000 & 9 & 2 & 9 & 2 & 3 & 5 & 11 & 10 & 4 & 5 & 6 & 5 & 6 & 12 & 11 \\
\hline
\end{tabular}




\section{Suyi Mao et al.}

volume (i.e. 3,000 vph, 5,000 vph and 10,000 vph) and eight different DMS distances (i.e. $400 \mathrm{~m}, 600 \mathrm{~m}, 1,000 \mathrm{~m}, 1,400 \mathrm{~m}$, $1,800 \mathrm{~m}, 2,200 \mathrm{~m}, 2,600 \mathrm{~m}$ and 3,000 m) are considered. The following three examples demonstrate the lane-changing scenarios in different experiments.

One of the cases is shown in Figure 6, which represents the distance from DMS and work zone is $400 \mathrm{~m}$ and the traffic volume is 5,000 vph. Because the distance between DMS and work zone is very short, according to the results, it is inferred that all the drivers will change lane immediately when they see information displayed on DMS.

One of the cases shown in Figure 7, which represents the distance from DMS and work zone, is $600 \mathrm{~m}$ and the traffic volume is $5,000 \mathrm{vph}$. Because the distance between DMS and work zone is short, according to the results, it can be inferred that more than $90 \%$ of drivers will change lane when they have seen information displayed on DMS as soon as possible.

As it is shown in Figure 8, when the distance between DMS and work zone is more than $1 \mathrm{~km}$, it was assumed that drivers are divided into three categories. Some drivers will change lanes immediately and some drivers will change lanes between DMS and $200 \mathrm{~m}$ from the work zone. And the others will change lanes just before the work zone. Because there is a traffic congestion, there are not enough opportunities for drivers to change lanes. Then, the lane change ratio of the subinterval close to the work zone is still high.

Experiment 2: In the advanced environment with $\mathrm{CV}, \mathrm{CVs}$ that can receive traffic information from in-vehicle warning device earlier than manually driven vehicles can only receive traffic information from DMS. It is assumed that the construction warning of work zone is sent to CVs by the roadside device within $600 \mathrm{~m}$ ahead of DMS. Therefore, CV drivers were divided into more categories and some will change lanes ahead of the DMS. Figure 9 displays one of the simulation scenarios when $\mathrm{CV}$ and $\mathrm{MV}$ are mixed in the road network.

The lane change ratios of manual vehicles (MVs) refer to the experimental results of the optimal lane change ratios. A new indicator named average safe lane-changing probability (ASLP), which reflects the probability of making a safe lane change maneuver in the subinterval, was proposed.

Figure 6 Distance between DMS and work zone is $400 \mathrm{~m}$ (5,000 vph)

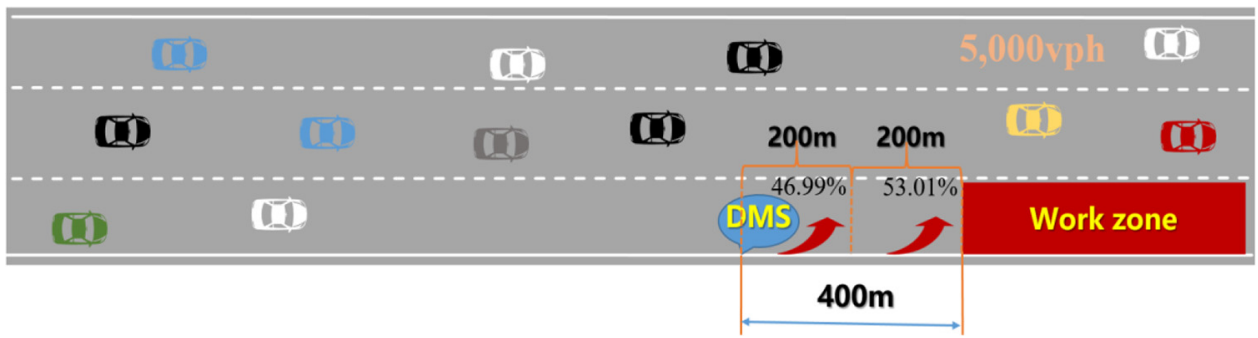

Figure 7 Distance between DMS and work zone is $600 \mathrm{~m}$ (5,000 vph)

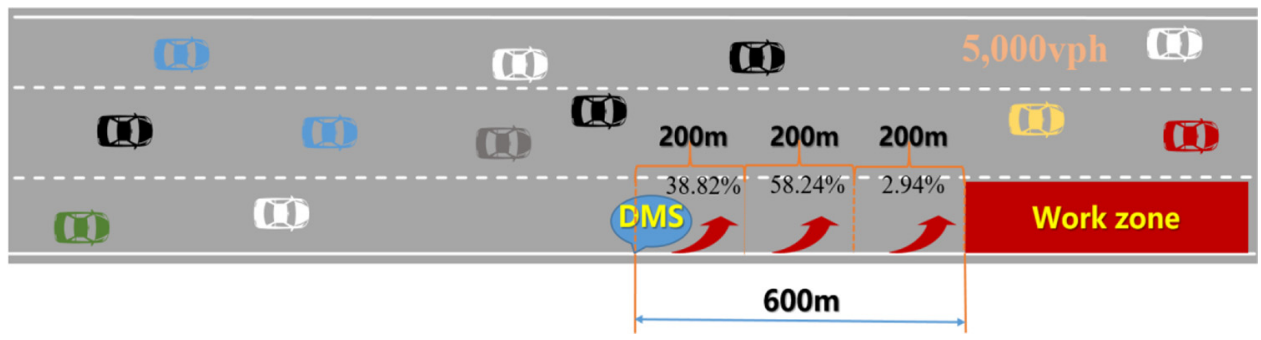

Figure 8 Distance between DMS and work zone is $1 \mathrm{~km}$ (5,000 vph)

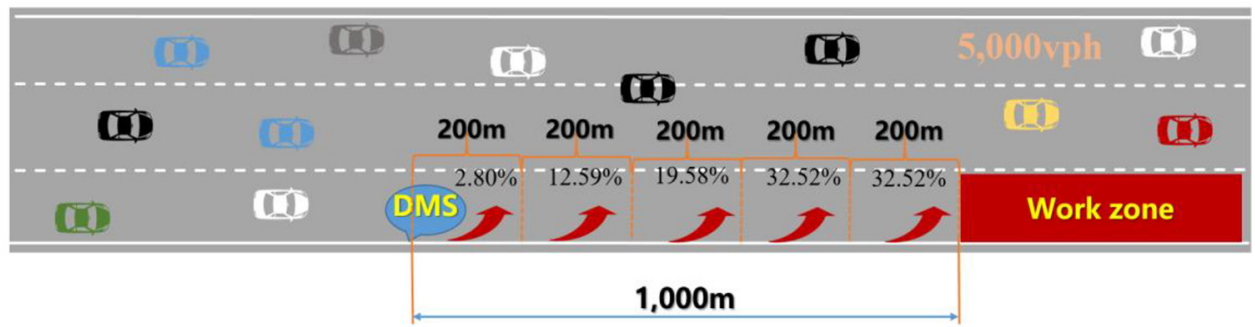


The calculation formula of $A S L P_{\text {subinterval }}$ is specified as follows:

$$
\begin{gathered}
A S L P_{\text {sub-interval }}=\frac{\sum_{i=1}^{n} f\left(s_{i t}\right)}{n} \\
f\left(s_{i t}\right)= \begin{cases}0 & T T C_{\text {approximation }}<1.5 \\
1 & T T C_{\text {approximation }}>=1.5\end{cases} \\
T T C_{\text {approximation }}=\frac{x_{L}-x_{F}+v_{L} * 5-v_{F} * 5}{v_{F-} v_{L}}
\end{gathered}
$$

where $A S L P_{\text {subinterval }}$ is the ASLP of a subinterval and $i$ is the number of vehicle whose active lane is the right lane. In this paper, there is only the possibility that vehicles in the right lane will change lanes because of the work zone layout. $f\left(s_{i t}\right)$ is the safe lane-changing probability of a vehicle numbered $i$ in the subinterval $s$ at time t. $n$ is the total number of vehicles. $x_{L}$ is abscissa value of the leading vehicle in the right lane; $x_{F}$ is abscissa value of the following vehicle in the middle lane that is closest to the leading vehicle and it is in the middle lane; it is assumed that the leading vehicle's lane changing maneuver will last $5 \mathrm{~s}$ averagely; $v_{F}$ is velocity of the following vehicle; and $v_{L}$ is velocity of the leading vehicle.

It is assumed that vehicles whose active lane is the right lane change lanes in the subinterval and calculate the $T T C_{\text {approximation }}$ between these vehicles and the nearest vehicles close to them in the middle lane. If $T T C_{\text {approximation }}$ is less than $1.5 \mathrm{~s}$, the value of $f\left(s_{i t}\right)$ is equal to 0 , which indicates that it is not safe for the vehicle to change lanes in the subinterval. If $T T C_{\text {approximation }}$ is more than $1.5 \mathrm{~s}$, the value of $f\left(s_{i t}\right)$ is equal to 1 , which indicates that it is safe for the vehicle to change lanes in the subinterval. According to the results of optimal lane change ratio, in the beginning of the experiment, all MV's data (location, speed, etc.) were collected to calculate the initial ASLP value of every subinterval. The value of ASLP in each subinterval is used as the reference standard for the recommended lane-changing ratio for $\mathrm{CV}$. The bigger the value of $A S L P_{\text {subinterval }}$ is, the higher the lane-changing ratio in the subinterval will be. It is assumed that CV's compliance rate is $100 \%$. In the first loop, the lane-changing ratio referred to the $A S L P_{\text {subinterval }}$ of the simulation scenario whose traffic flow composition is full of $M V$. And in the second loop, the recommended lane-changing ratio referred to the $A S L P_{\text {subinterval }}$ calculated in the first loop is send to CV. After simulation, the difference of $A S L P_{\text {subinterval }}$ between this loop and the previous loop is calculated. The loop continues until this value is small enough. For different $\mathrm{CV}$ market penetration rates scenarios, the composition of lane changing ratios was recalculated and the market penetration rates of $\mathrm{CV}$ are increased successively.

Different market penetration rates of $\mathrm{CV}$ (i.e. $0 \%, 30 \%$, $50 \%, 70 \%$ and $100 \%$ ) were considered. There may be a phenomenon of information oversaturation because $\mathrm{CV}$ drivers will also see DMS. It was assumed that CV drivers will not respond to DMS, they only respond to the advanced roadside device. According to the results of Experiment 1, only one of the safest DMS distance scenarios was used in Experiment 2 under each traffic volume condition because it takes a long time to run an experiment.

\section{Results}

Experiment 1: Figures 10-12 exhibit the simulation results of different traffic volume scenarios in the traditional environment, respectively. Results represent the safety and operational effects of placing DMS in different location.

Figure 10 shows that it is more effective when the DMS is placed at $3 \mathrm{~km}$ in the upstream from the work zone considering efficiency and safety. The value of $Q T_{\text {mean }}$ and $D T_{\text {mean }}$ is close to 0 . Because traffic volume of this scenario is small, it indicates that the traffic is very smooth. When under the condition of free flow, the distance between DMS and work zone has little influence on the efficiency of the whole traffic environment (i.e. average delay time, average queue time, average speed and travel time). This is because there are many opportunities to change lanes, thus it is safer to place DMS far from work zone. It can avoid the concentration of the lane-changing behavior that may cause more lateral conflicts. The lane-changing ratio is even in the interval, which indicates that drivers merge at different subintervals evenly. When to change lanes depends on drivers' characteristics and their driving habit. However, if the DMS placing distance is too short from the work zone, drivers would not have enough space to change lanes, which may lead to drivers' aggressive merging maneuver, and it might cause more conflicts when they are merging out from the right lane.

Figure 11 presents that it is more effective when the DMS is placed $2.2 \mathrm{~km}$ upstream of the work zone considering safety. When under the condition of saturated flow meaning that the traffic is no longer smooth, long-distance DMS placed from the work zone may lead to lane-changing behaviors ahead of time. However, it is not ideal for current traffic conditions to meet the requirements of completing a lane-changing maneuver, which is easy to cause more lateral conflicts between vehicles. Compared with the free flow, the safest distance between DMS and work zone is shorter. Correspondingly, when the distance

Figure 9 Schematic of CV and MV are mixed in the road network

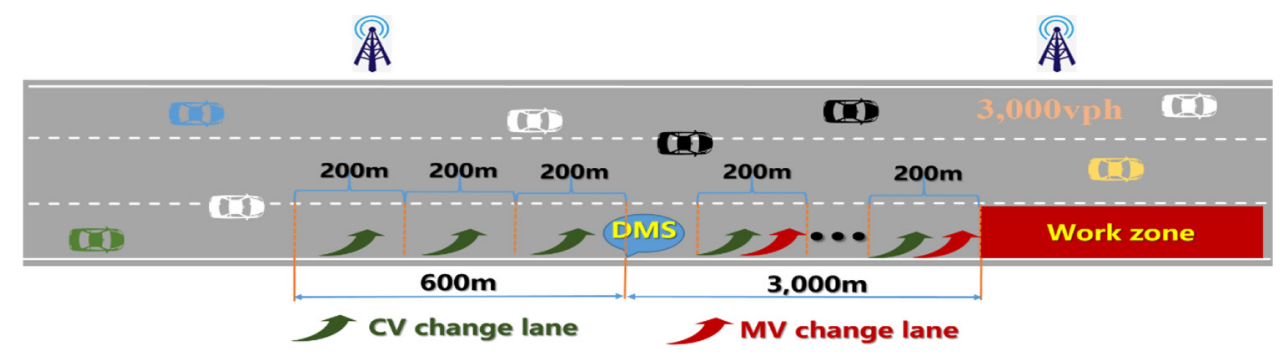


become longer, the negative influence on the safety and efficiency of the whole traffic system is more obvious. According to the results, a reasonable inference that it may lead to traffic chaos if informed drivers too early when there is a traffic congestion can be made.

Figure 12 presents that when the DMS is placed $1.0 \mathrm{~km}$ upstream of the work zone, travel time is smallest, whereas $N_{T T C \leq 1.5}$ is not the smallest. Because of the heavy traffic, drivers will not have sufficient opportunities to change lanes and they will keep driving forward until they have an opportunity to change lanes, which might eventually cause traffic congestion near the work zone. The safest distance DMS placed from the work zone is $1.8 \mathrm{~km}$ in this scenario. Similarly, compared with the scenario whose traffic volume is $5,000 \mathrm{vph}$, the safest distance DMS placed from the work zone is also shorter.

Therefore, it can be concluded from the above results that when traffic is relatively smooth, the location of DMS should be far from the work zone, which not only improves the safety level of the whole traffic system, but also prevents affecting the efficiency of the whole traffic system. The higher the value of traffic volume is, the more obvious negative influence of longdistance DMS placed from the work zone will have on the efficiency and safety of the whole traffic system. The best distance DMS placed from the work zone should be appropriately shortened when there is a traffic congestion.

Figure 13 exhibits the safety and efficiency effects when DMS was placed at the safest distance from the work zone

Figure 10 Safety and efficiency effects of DMS at 3,000 vph

\section{Indicators' variation of different scenarios (3,000vph)}

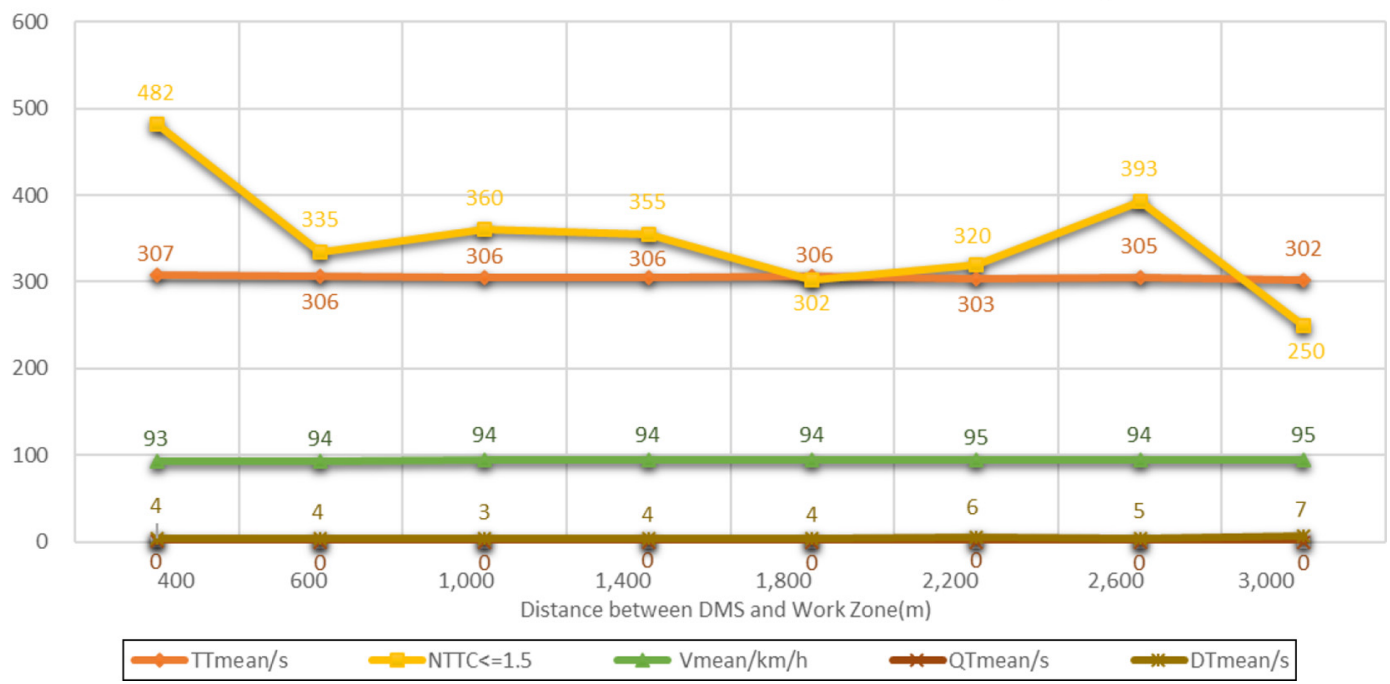

Figure 11 Safety and efficiency effects of DMS at 5,000 vph

\section{Indicators' variation of different scenarios $(5,000 \mathrm{vph})$}

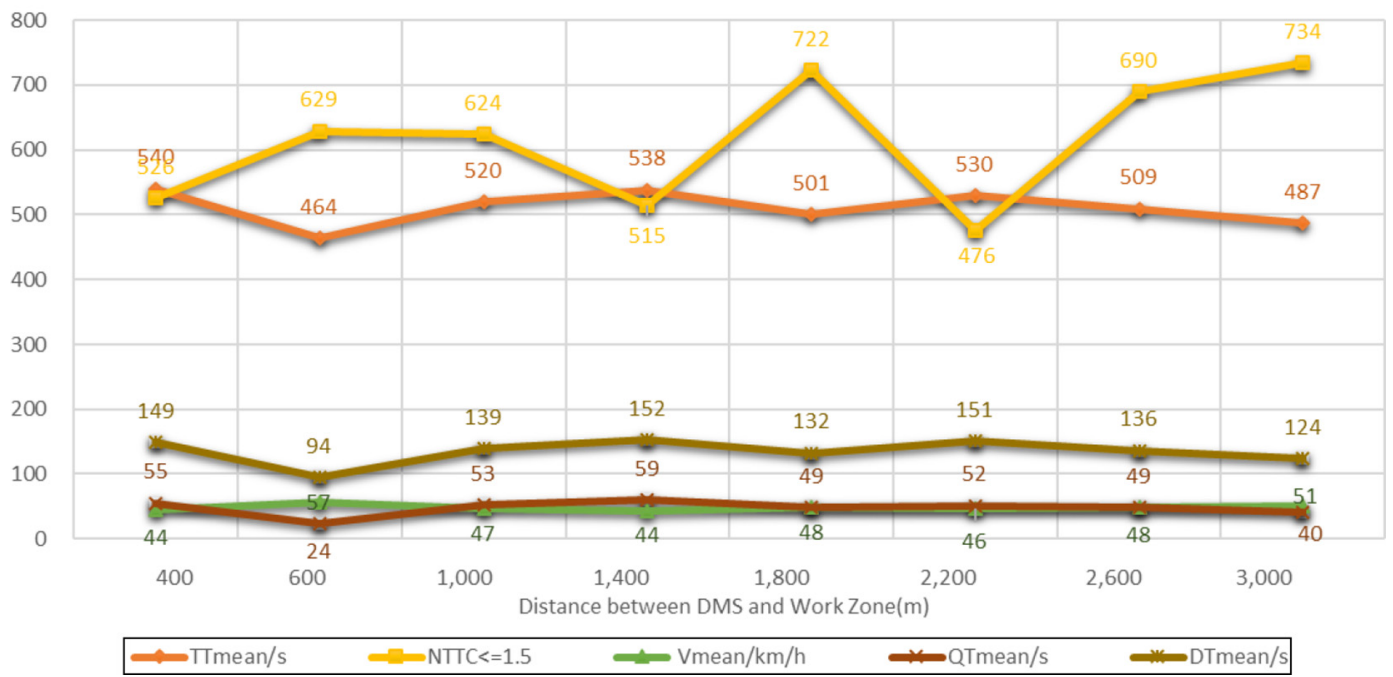


under different traffic volume scenarios. The figure shows that as traffic volume increases, crash risks increases, whereas traffic efficiency of the whole traffic system decreases, which is consistent with the common sense. Moreover, when traffic volume increases, the safest distance between DMS and work zone is shorter, which is owing to the interaction between efficiency and safety level of the whole traffic system.

Experiment 2: Figures 14-16 exhibit the safety and efficiency effects of different market penetration rates at different traffic volume scenarios (i.e. 3,000 vph, 5,000 vph and 10,000 vph) in the mixed advanced traffic environment. Traffic efficiency indicators and $N_{T T C \leq 1.5}$ representing the number of conflicts have been calculated.

As shown in the Figure 14, the value of indicators representing the efficiency of the whole traffic system varies slightly when traffic volume is at 3,000 vph. It is worth noting that the value of $Q T_{\text {mean }}$ and $D T_{\text {mean }}$ is close to 0 , which is same with the results in Figure 10. As mentioned earlier, the traffic volume of these two scenarios is small. There is almost no queuing and delay. However, the value of
$N_{T T C \leq 1.5}$ varies greatly. Obviously, when the MPR is $100 \%$, the value of $N_{T T C \leq 1.5}$ is smallest, which indicates that the number of traffic conflicts under the environment of full $\mathrm{CV}$ is safest. The popularization of $\mathrm{CV}$ is a gradual process and different $\mathrm{MPR}$ of $\mathrm{CV}$ has to be considered during this period. With the increase of the MPR of $\mathrm{CV}$, the traffic safety risks show volatility. When the MPR of CV is $50 \%$, the value of $N_{T T C \leq 1.5}$ is greater than the value of $N_{T T C \leq 1.5}$ under full MV environment, which revealed that the MPR of $\mathrm{CV}$ and traffic safety risks do not conform to a simple linear relationship.

Figure 15 and 16 show that the safety and efficiency effect under different MPR when the traffic volume is heavy. When the MPR of CV is $100 \%, N_{T T C \leq 1.5}$ is smallest, which indicates the scenarios is safest. With the increase of MPR of CV, the number of traffic conflicts increase on the contrary in the beginning. It can be inferred that when there is serious traffic congestion, the advanced advisory system cannot work well. Because of the poor traffic condition, there would be almost no opportunities for drivers to change lanes even though they receive the advanced information.

Figure 12 Safety and efficiency effects of DMS at 10,000 vph

\section{Indicators' variation of different scenarios $(10,000 \mathrm{vph})$}

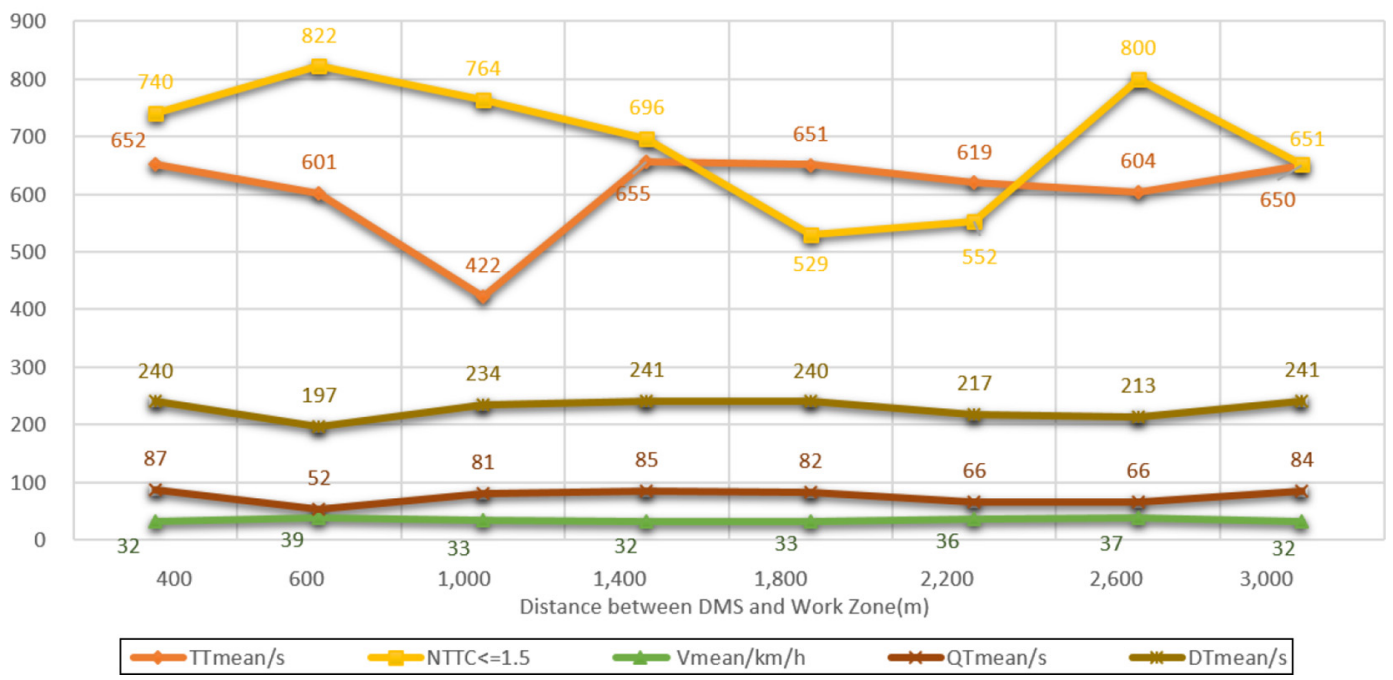

Figure 13 Safety and efficiency effects of DMS at different traffic volume

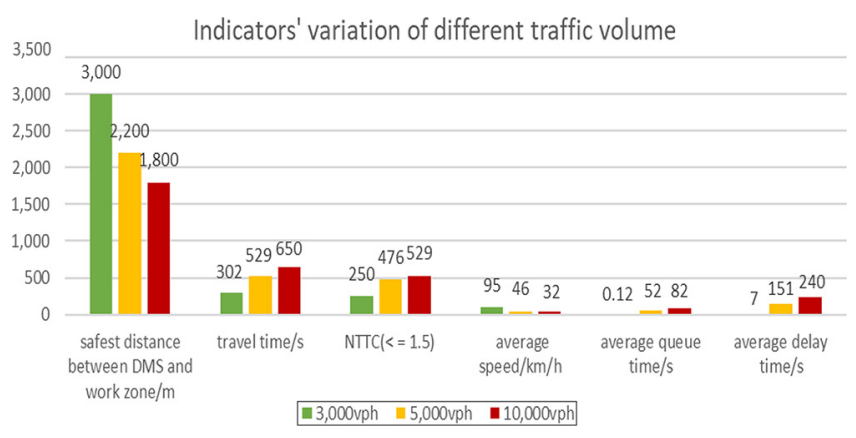

\section{Discussions of the results}

With the rapid development of information and communication technologies, more and more advanced equipment is put into application. The study identified that the improvement of efficiency and safety effects in the freeway construction scene by using advanced traffic information delivery approaches. In the traditional environment using DMS to deliver warning information, the position and content influence drivers' compliance rate. The safest distance DMS placed from the work zone is different under different traffic condition (i.e. smooth, congested and supersaturated). When traffic is relatively smooth, the location of DMS should be far from the work zone, which can not only improve the safety level of the whole traffic system, but also avoid affecting the 
Figure 14 Safety and efficiency effects under different MPR at 3,000 vph

Indicators' variation under different MPR of CV at 3,000vph

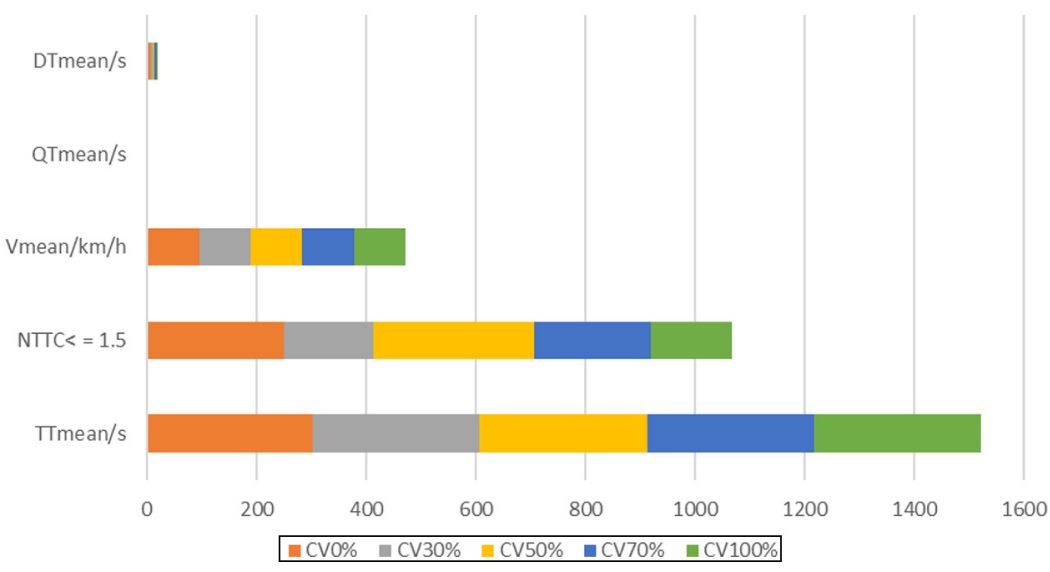

Figure 15 Safety and efficiency effects under different MPR at 5,000 vph

Indicators' variation under different MPR of CV at 5,000vph

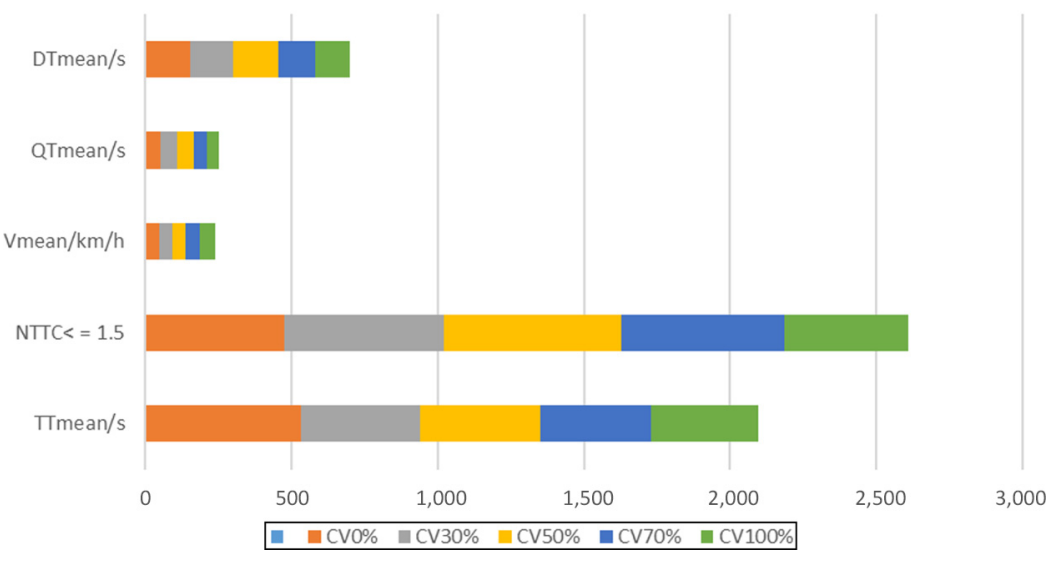

Figure 16 Safety and efficiency effects under different MPR at 10,000 vph

Indicators' variation under different MPR of CV at 10,000vph

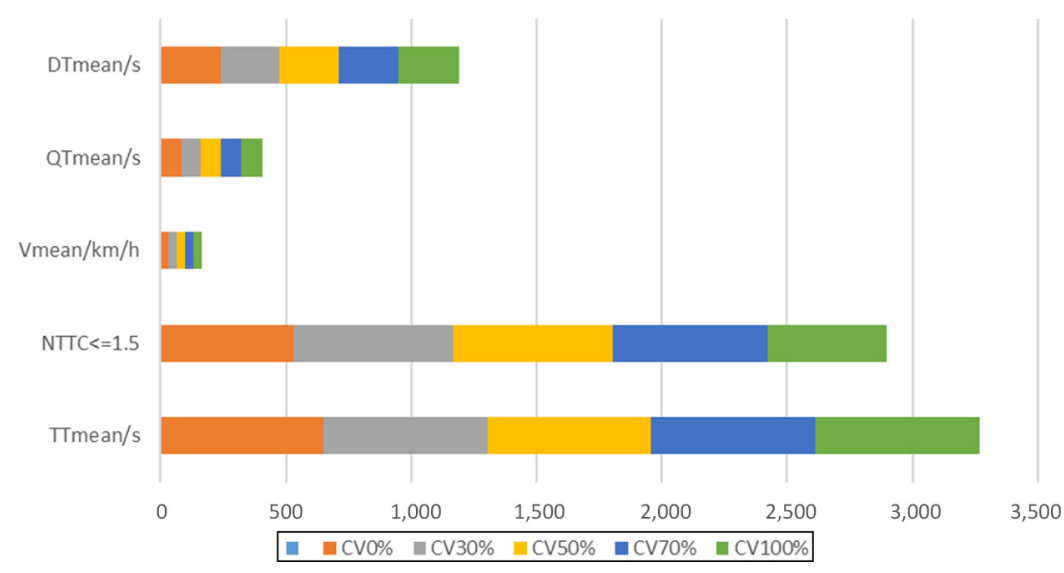


efficiency of the whole traffic system. However, the higher the value of traffic volume is, the more obvious negative influence of long distance DMS placed from the work zone will have on the efficiency and safety of the whole traffic system. It can be inferred that drivers did not have much opportunities to change lanes because of the bad traffic condition. Overlong distance for lane changing will cause later conflicts of vehicles on the contrary. Currently, many researchers agree that both efficiency and safety are of the top priority in the transportation system. A balance between pursuing efficiency and considering safety should been found. The above results show that advanced communication technology in the $\mathrm{CV}$ environment will greatly improve traffic efficiency. However, the safety effects near the work area does not conform to a simple rule, which may be affected by the structure of the road network, traffic conditions, the operation of advanced technology applications, the complex driving behaviors of drivers and so on.

Furthermore, more detailed traffic information provided in the $\mathrm{CV}$ environment may lead to excessive information, which might negatively affect drivers' behavior. From the experimental results above, with the increase of the MPR of $\mathrm{CV}$, the traffic safety risks show volatility. When the MPR of $\mathrm{CV}$ is $50 \%$, the value of $N_{T T C \leq 1.5}$ is more than it under total MV environment, which revealed that the MPR of CV and traffic safety risks do not conform to a simple linear relationship. We can infer that when $\mathrm{CV}$ drivers receive information earlier than non-CV drivers, CV drivers will change lanes earlier than them, there will cause more traffic conflicts because of the mixed traffic flow conditions. Because of the increased lateral behaviors, the risk of collision will increase simultaneously. When the MPR of $\mathrm{CV}$ is $50 \%$, the level of confusion is highest. To improve the efficiency and safety of the whole system, some strategies should be proposed and applied to solve the problems, which occur because of traffic component confusion. Moreover, when the traffic conditions become worse, the advanced advisory system cannot work well. The strategy giving advance notice at appropriate position for dispersing volume to ensure good traffic condition can be used. Therefore, making good use of traditional and advanced advisory system together is necessary.

\section{Conclusions}

Existing ITS applications alone are still great tools to improve capacity and safety. DMS has played a key role to deliver traffic and event (e.g. work zone) information to drivers and the drivers can be prepared for the upcoming traffic situations. Nevertheless, DMS information is provided in the same way to all drivers. Some drivers will react very quickly; others might not. When traffic efficiency and safety are taken into consideration together, the most beneficial distance DMS placed from the work zone should be appropriately shorten when there is traffic congestion. Specific traffic information provided in the $\mathrm{CV}$ environment will improve the efficiency and safety of the whole transportation system. The state of $\mathrm{CV}$ and manually driven vehicle mixed in the road network is expected to last for decades. Make full use of the existing ITS system and integrate it with the upcoming advanced $\mathrm{CV}$ technologies, which is more beneficial to maintain a healthy and stable traffic environment. It is worth noting that the MPR of $\mathrm{CV}$ and traffic safety risks do not conform to a simple linear relationship. The introduction of $\mathrm{CV}$ into the whole traffic system is a complicated issue. The effect that CV will have on the whole traffic system is an urge key scientific problem that needs to be solved.

It is worthy to note several limitations to this paper. First, excessive information might be provided to some drivers because CV drivers will also see DMS information. Such information overload should be considered in follow-up studies. Second, because this study used only microsimulation, results do not reflect the real-world drivers' reactions to DMS and $\mathrm{CV}$ warning messages. And the objective of this research mainly focuses on V2I links by considering the connection between infrastructure and vehicles under connected environment. vehicle-to-vehicle links will be considered in the future study. A series of driving simulator experiments need to be conducted to capture the real driving behaviors so as to investigate the unresolved related issues. Human machine interface needs be used to simulate the process of in-vehicle warning information delivery. Third, the validation of the simulation model was not conducted because of the data limitation. Real data of each scenario should be collected for validation and deep analysis to ensure the credibility of the experiment results.

\section{References}

Abdulsattar, H., Mostafizi, A. and Wang, H. (2018), "Surrogate safety assessment of work zone rear-end collisions in a connected vehicle environment: agent-based modeling framework", fournal of Transportation Engineering, Part A: Systems, Vol. 144 No. 8, p. 4018038

Ardeshiri, A. and Jeihani, M. (2014), "A speed limit compliance model for dynamic speed display sign", fournal of Safety Research, Vol. 51, pp. 33-40.

Bella, F. (2005), "Validation of a driving simulator for work zone design", Transportation Research Record: Fournal of the Transportation Research Board, Vol. 1937 No. 1, pp. 136-144.

Blackman, R., Debnath, A. and Haworth, N. (2014), "Work zone items influencing driver speeds at roadworks: worker, driver and expert perspectives", In Proceedings of the 2014 Australasian Road Safety Research, Policing and Education Conference: Australasian College of Road Safety (ACRS), pp. 1-11.

Brewer, M.A., Pesti, G. and Schneider, I.V., W. (2006), "Improving compliance with work zone speed limits: effectiveness of selected devices", Transportation Research Record: Fournal of the Transportation Research Board, Vol. 1948 No. 1, pp. 67-76.

Carsten, O. and Jamson, A.H. (2011), "Driving simulators as research tools in traffic psychology", In Handbook of Traffic Psychology, Academic Press, pp. 87-96.

Debnath, A.K., Blackman, R. and Haworth, N. (2015), "Common hazards and their mitigating measures in work zones: a qualitative study of worker perceptions", Safety Science, Vol. 72, pp. 293-301.

Dudek, C.L. (2004), "Changeable message sign operation and messaging handbook (no. FHWA-OP-03-070). United States. Federal highway administration". 
Genders, W. and Razavi, S.N. (2016), "Impact of connected vehicle on work zone network safety through dynamic route guidance", Fournal of Computing in Civil Engineering, Vol. 30 No. 2, p. 4015020

Hassan, H.M., Abdel-Aty, M.A., Choi, K. and Algadhi, S.A. (2012), "Driver behavior and preferences for changeable message signs and variable speed limits in reduced visibility conditions”, Fournal of Intelligent Transportation Systems, Vol. 16 No. 3, pp. 132-146.

Jones, S.L. and Thompson, M.W. (2003), State of the Practice for Displaying Non-Traffic Related Messages on Dynamic Message Signs, University Transportation Center for AL, Birmingham, AL.

Koutsopoulos, H.N., Polydoropoulou, A. and Ben-Akiva, M. (1995), "Travel simulators for data collection on driver behavior in the presence of information", Transportation Research Part C: Emerging Technologies, Vol. 3 No. 3, pp. 143-159.

Lajunen, T. and Summala, H. (2003), "Can we trust selfreports of driving? Effects of impression management on driver behaviour questionnaire responses", Transportation Research Part F: Traffic Psychology and Behaviour, Vol. 6 No. 2, pp. 97-107.

Mohammadi, M.A. Bham, G.H. and Khazraee, H. (2011), "An exploratory study of speed limit compliance in Missouri work zones", In Transportation and Development Institute Congress 2011: Integrated Transportation and Development for a Better Tomorrow, pp. 1216-1225.

National Work Zone Safety Information Clearinghouse (2019), "Work zone fatal crashes and fatalities", available at: www. workzonesafety.org/crash-information/work-zone-fatal-crashesfatalities/\#national, (accessed 30 October 2019).

Olia, A., Genders, W. and Razavi, S.N. (2013), "Microsimulation-based impact assessment of the vehicleto-vehicle (V2V) system for work zone safety", GEN, Vol. 211, p. 1.

Olson, R.L. Hanowski, R.J. Hickman, J.S. and Bocanegra, J. (2009), "Driver distraction in commercial vehicle operations (no. FMCSA-RRT-09-042). United States. Department of transportation. Federal motor carrier safety administration".

Rahman, M.S., Abdel-Aty, M., Wang, L. and Lee, J. (2018), "Understanding the highway safety benefits of different approaches of connected vehicles in reduced visibility conditions", Transportation Research Record: Fournal of the Transportation Research Board, Vol. 2672 No. 19, pp. 91-101.

Rahman, M.M., Strawderman, L., Garrison, T., Eakin, D. and Williams, C.C. (2017), "Work zone sign design for increased driver compliance and worker safety", Accident Analysis $\mathcal{E}$ Prevention, Vol. 106, pp. 67-75.
Schofer, J.L., Khattak, A. and Koppelman, F.S. (1993), "Behavioral issues in the design and evaluation of advanced traveler information systems", Transportation Research Part C: Emerging Technologies, Vol. 1 No. 2, pp. 107-117.

Shakouri, M., Ikuma, L.H., Aghazadeh, F., Punniaraj, K. and Ishak, S. (2014), "Effects of work zone configurations and traffic density on performance variables and subjective workload", Accident Analysis \& Prevention, Vol. 71, pp. 166-176.

Strawderman, L., Huang, Y. and Garrison, T. (2013), "The effect of design and placement of work-zone warning signs on driver speed compliance: a simulator-based study", IIE Transactions on Occupational Ergonomics and Human Factors, Vol. 1 No. 1, pp. 66-75.

Teizer, J., Allread, B.S., Fullerton, C.E. and Hinze, J. (2010), "Autonomous pro-active real-time construction worker and equipment operator proximity safety alert system", Automation in Construction, Vol. 19 No. 5, pp. 630-640.

Van Der Horst, R. and Hogema, J. (1993), "Time-to-collision and collision avoidance systems", In Proceedings of the 6th ICTCT workshop: Safety Evaluation of Traffic Systems: Traffic Conflicts and other Measures, pp. 109-121.

Wåhlberg, A., Dorn, L. and Kline, T. (2011), "The Manchester driver behaviour questionnaire as a predictor of road traffic accidents", Theoretical Issues in Ergonomics Science, Vol. 12 No. 1, pp. 66-86.

Wang, L., Abdel-Aty, M. and Lee, J. (2017), "Implementation of active traffic management strategies for safety on congested expressway weaving segments", Transportation Research Record: Fournal of the Transportation Research Board, Vol. 2635 No. 1, pp. 28-35.

Weng, J. and Meng, Q. (2012), "Effects of environment, vehicle and driver characteristics on risky driving behavior at work zones", Safety Science, Vol. 50 No. 4, pp. 1034-1042.

Whitmire, II., J., Morgan, J.F., Oron-Gilad, T. and Hancock, P.A. (2011), "The effect of in-vehicle warning systems on speed compliance in work zones", Transportation Research Part F: Traffic Psychology and Behaviour, Vol. 14 No. 5, pp. 331-340.

\section{Further reading}

Allpress, J.A. and Leland, L.S. (2010), "Reducing traffic speed within roadwork sites using obtrusive perceptual countermeasures", Accident Analysis \& Prevention, Vol. 42 No. 2, pp. 377-383.

\section{Corresponding author}

Jaeyoung Lee can be contacted at: lizaining@csu.edu.cn 